
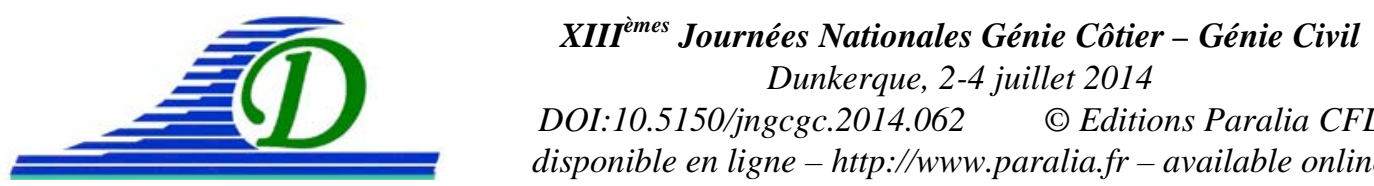

DOI:10.5150/jngcgc.2014.062 @ Editions Paralia CFL

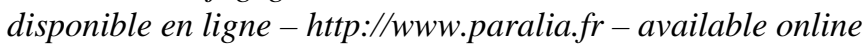

\title{
Détermination de la position du trait de côte à partir de levés topographiques LiDAR aéroportés
}

\author{
Adrien CRAPOULET ${ }^{1}$, Arnaud HEQUETTE ${ }^{1}$, Patrice BRETEL ${ }^{2}$, \\ Franck LEVOY ${ }^{2}$
}

1. Laboratoire d’Océanologie et de Géosciences, UMR CNRS 8187, Université du Littoral Côte d’Opale, 32 Ave Foch, 62930 Wimereux, France. adrien.crapoulet@univ-littoral.fr ; arnaud.hequette@univ-littoral.fr

2. Laboratoire "Morphodynamique Continentale et Côtière", UMR CNRS 6143, Université de Caen Basse Normandie, 24 Rue des tilleuls, 14000 Caen cedex, France.

\section{Résumé :}

La position de différents indicateurs de trait de côte a été déterminée à l'aide de modèles numériques de terrain générés à partir des données LiDAR. Un premier trait de côte ( $\left.\mathrm{TC}_{\mathrm{PHMA}}\right)$ a été déterminé en utilisant la cote altimétrique des plus hautes mers astronomiques (PHMA). Une deuxième position de trait de côte $\left(\mathrm{TC}_{\mathrm{grad}}\right)$ a été définie en fonction des variations de pente sur le haut de plage en calculant un gradient de pentes à partir de modèles numériques de terrain. Ces traits de côte ont été comparés à un trait de côte déterminé in situ par DGPS, correspondant à la limite morphologique (rupture de pente) entre le haut de plage et les dunes côtières ainsi qu'à un trait de côte défini à partir de photographie aérienne correspondant à la limite de la végétation dunaire ou à une limite morphologique. Les résultats montrent que le trait de côte $\mathrm{TC}_{\text {grad }}$ est celui qui correspond le plus étroitement aux positions de trait de côte relevées directement sur le terrain. Des mesures de l'évolution de la ligne de rivage ont également montré que le $\mathrm{TC}_{\mathrm{PHMA}}$ est dans la très grande majorité des cas situé à l'avant de la position du $\mathrm{TC}_{\text {grad }}$ là où la dune a connu une progradation vers le large. La position du TC $\mathrm{THMA}_{\text {PHAr rapport au }}$ TC $_{\text {grad }}$ pourrait par conséquent constituer un bon indicateur de la tendance évolutive de la ligne de rivage le long des littoraux dunaires.

Mots-clés : Trait de côte, LiDAR, DGPS, Plages macrotidales, Cordon dunaire, NordPas-de-Calais.

\section{Introduction}

Pendant les dernières décennies, la technologie LiDAR (light detection and ranging) a connu un essor considérable dans les études consacrées à l'étude de la morphologie et de l'évolution de la zone côtière (BROCK et al., 2009 ; LEVOY et al., 2013), cette technologie permettant d'obtenir une restitution fine de la topographie (marge d'erreur verticale $< \pm 10 \mathrm{~cm}$ sur sol nu) sur de vastes superficies. Les modèles numériques de terrain issus de données topographiques LiDAR sont en outre souvent utilisés pour déterminer des bilans sédimentaires (DERONDE et al., 2008) ainsi que les zones à 
risques d'érosion et de submersion le long du littoral (STOCKDON et al., 2009). Dans le cadre de telles études, il est essentiel de pouvoir déterminer de façon précise la position de l'objet que l'on définit comme indicateur du trait de côte si l'on veut être en mesure de suivre son évolution dans le temps. La détermination de la position du trait de côte demeure cependant un exercice difficile, car elle peut être établie à partir de critères différents qui peuvent être d'ordre morphologique, hydrographique ou même biologique (e.g., limite de végétation) (STEPHAN \& SUANEZ, 2004 ; BOAK \& TURNER, 2005; BRETEL et al., 2013). L'objectif de l'étude présentée ici est d'évaluer la méthode la plus appropriée pour déterminer la position du trait de côte à partir de données topographiques LiDAR en comparant les résultats obtenus avec d'autres méthodes de localisation du trait de côte.

\section{Zones d'étude}

Les sites d'étude choisis correspondent à deux plages sableuses à barres intertidales de la côte du Nord-Pas-de-Calais (NPDC) situées à Oye-Plage (réserve naturelle du Platier d'Oye) et à l'est de Dunkerque (figure 1). Ces deux sites font face à la mer du Nord et sont soumis à un régime de vents et de houles dominants du SO. Associé à une dissymétrie de la marée responsable d'une dominance des courants de flot par rapport aux courants de jusants, ce régime dominant provoque un transport sédimentaire vers l'est (SIPKA \& ANTHONY, 1999). L'autre direction majeure de vents et de houle est le secteur nord qui, de par sa direction frontale à la côte, peut provoquer des épisodes érosifs (RUZ et al., 2009).

Le premier site, situé entre Dunkerque et la frontière belge, est caractérisé par une plage de sables fins $\left(D_{50}=0,2 \mathrm{~mm}\right)$, de 350 à $450 \mathrm{~m}$ de largeur, ayant une pente moyenne d'environ $0,014(\tan \beta)$. Cette plage est associée à deux massifs dunaires d'environ $700 \mathrm{~m}$ de largeur, nommés dune Dewulf et dune Marchand, présentant des dunes paraboliques et un cordon bordier linéaire de 7 à $15 \mathrm{~m}$ de hauteur. Des levés topographiques réguliers du haut de plage et du cordon bordier de la dune Dewulf ont montré que le front dunaire pouvait reculer de 1 à $2 \mathrm{~m}$ pendant une seule tempête, l'analyse de photographies aériennes diachroniques a révélé que dans l'ensemble ce secteur côtier est relativement stable depuis le milieu des années 90 (MASPATAUD et al., 2011). Le régime tidal, semi-diurne, est de type méso à macrotidal, avec un marnage moyen de mortes eaux de 3,5 m et de 5,45 m en vives eaux.

Le second site, le platier d'Oye, se situe entre Calais et Gravelines, sur la rive ouest de l'embouchure endiguée du fleuve Aa. Située à l'est d'une plateforme sableuse d'environ 1 km de large, formée par accolement à la côte d'un banc sableux subtidal (HEQUETTE \& AERNOUTS, 2010), la plage du platier d'Oye est nettement moins large, de l'ordre de $300 \mathrm{~m}$. Composée également de sables fins $\left(D_{50}=0,24 \mathrm{~mm}\right)$, son système de barres sableuses intertidales est perturbé par la présence de quatre épis qui ont été installés afin d'essayer d'enrayer l'érosion qui a touché le cordon dunaire 


\section{XIII ${ }^{\text {èmes }}$ Journées Nationales Génie Côtier - Génie Civil \\ Dunkerque, 2-4 juillet 2014}

pendant les dernières décennies (AUBRY \& RUZ, 2008). Dans la partie centrale de la plage, le front du cordon dunaire a reculé d'environ $1 \mathrm{~m} / \mathrm{an}$ pendant les dernières décennies, tandis qu'aux extrémités ouest et est du site, deux flèches sableuses se sont développées et ont progressé pendant la même période. En raison d'une augmentation du marnage de la mer du Nord vers la Manche, l'amplitude de la marée est légèrement supérieure sur ce site, le marnage étant de 3,75 m et de 5,85 m en mortes eaux et vives eaux moyennes.

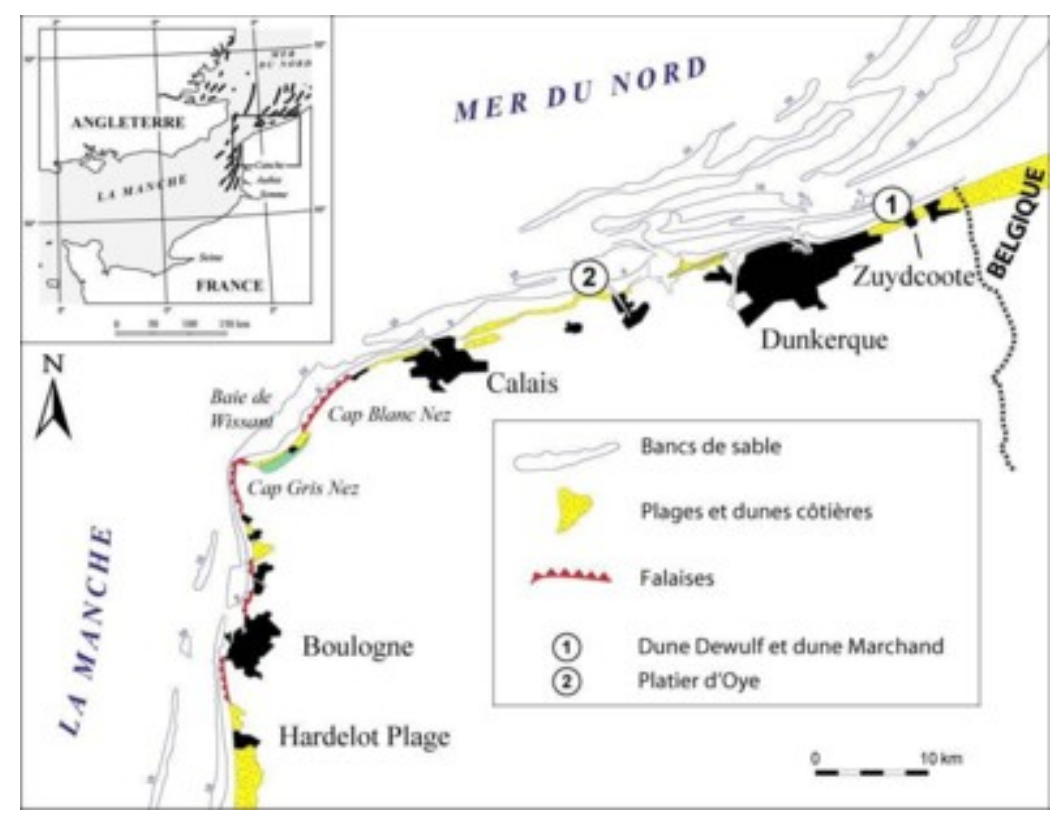

Figure 1. Localisation des sites d'étude.

\section{Méthodologie}

Pour calculer la cinématique du trait de côte, différentes techniques ont été utilisées, et ce à plusieurs pas de temps différents. La première technique employée consiste à tracer le trait de côte par suivi du pied de dune ou de la végétation dunaire à partir d'une photographie aérienne ortho-rectifiée et géo-référencée en utilisant un logiciel SIG (ArcGIS). Le pied de dune a été utilisé lorsqu'il a pu être identifié à l'aide de couples stéréoscopiques. En absence d'identification du pied de dune, ce qui a souvent été le cas dans les secteurs en voie de progradation, la limite de végétation a été retenue. Les photographies retenues correspondent aux années 1963, 1983, 2000, 2005 et 2009, permettant ainsi de calculer la cinématique du trait de côte sur près de cinquante ans.

La seconde technique employée consiste en une extraction du trait de côte à partir des données topographiques LiDAR. Les données utilisées sont issues de deux levés LiDAR complets de la zone littorale du Nord-Pas-de-Calais réalisés en 2008-2009 et en 2011. Ces jeux de données topographiques issues du LiDAR ont une marge d'erreur verticale de l'ordre de $\pm 10 \mathrm{~cm}$, cette précision étant moindre cependant en présence de 
végétation. Deux positions de trait de côte ont été déterminées à l'aide de modèles numériques de terrain (MNT) générés à partir des données LiDAR. Ces MNT sont obtenus par triangulation avec interpolation linéaire des données topographiques brutes à l'aide du logiciel Golden Software Surfer en appliquant un maillage de 0,5 m.

Le niveau des plus hautes marées astronomiques (PHMA) a été choisi pour définir une position de trait de côte ( $\left.\mathrm{TC}_{\mathrm{PHMA}}\right)$, ce qui correspond à la définition proposée par l'Organisation Hydrographique Internationale. Le trait de côte est donc obtenu dans ce cas par extraction de la courbe altimétrique correspondant à ce niveau de marée. Bien que présentant l'avantage de fortement réduire la marge d'erreur par rapport à la technique de suivi par photographie aérienne, le $\mathrm{TC}_{\mathrm{PHMA}}$ ne reflète pas nécessairement la réalité morphologique du trait de côte qui, dans le cas d'un cordon dunaire par exemple, correspondrait au pied de dune.

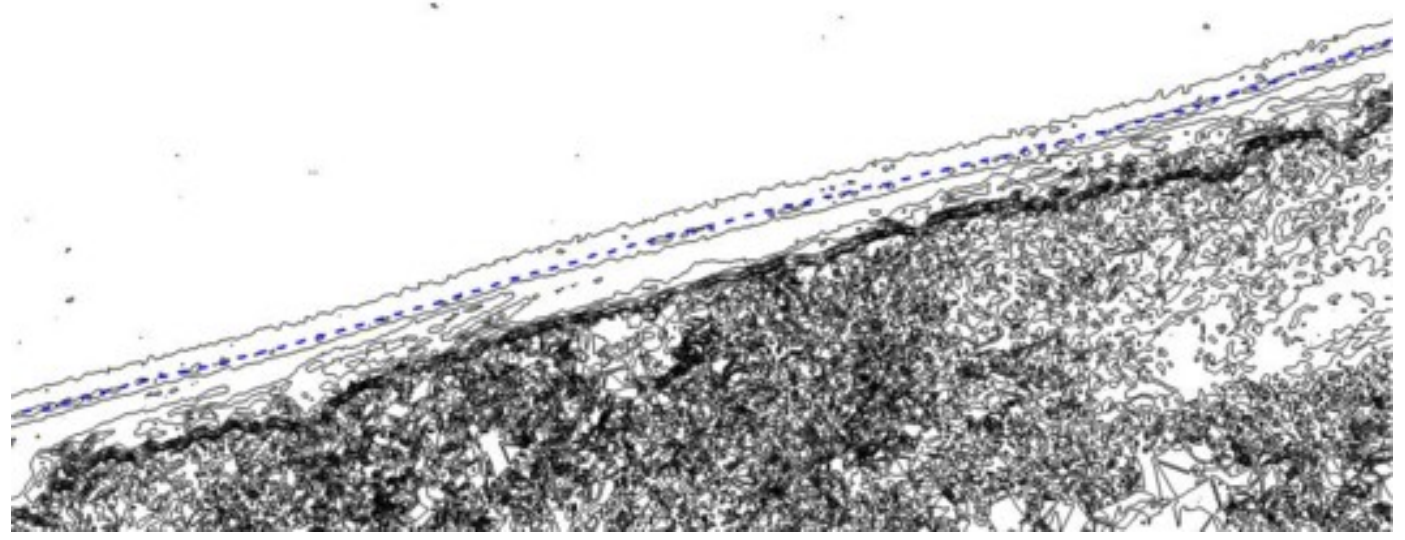

Figure 2. Carte d'isolignes de variation du gradient de pente sur le littoral du platier d'Oye obtenue à partir des données topographiques LiDAR 2011. La ligne en tirets correspond au TC

Une deuxième position de trait de côte $\left(\mathrm{TC}_{\mathrm{grad}}\right)$ a été définie en fonction des variations de pente sur le haut de plage en calculant un gradient de pentes (Eq.1) à partir des modèles numériques de terrain :

$\|\vec{g}\|=\sqrt{\left(\frac{\partial z}{\partial x}\right)^{2}+\left(\frac{\partial z}{\partial y}\right)^{2}}$

où $\vec{g}$ est le gradient, $z$ l'altitude, $x$ et $y$ les coordonnées de chaque point de calcul de la grille. La valeur du gradient est de 0 pour une surface horizontale et tend vers l'infini pour une pente s'approchant de la verticale. A partir de ce calcul, il est possible d'afficher une représentation des isolignes de variation du gradient de pente. Le cordon dunaire apparait alors sous la forme d'un net resserrement des isolignes, représentant une brusque élévation de la pente (figure 2). Le trait de côte choisi correspond alors à la $1^{\text {ère }}$ isoligne de ce resserrement et donc à une valeur de gradient de pente marquant la 


\section{XIII ${ }^{\text {èmes }}$ Journées Nationales Génie Côtier - Génie Civil \\ Dunkerque, 2-4 juillet 2014}

transition entre le haut de plage et la dune. Afin de valider cette méthode d'extraction du trait de côte par la méthode du gradient de pente, la position du $\mathrm{TC}_{\text {grad }}$ a été comparée à celle du trait de côte levé sur le terrain par suivi du pied de dune à l'aide d'un GPS différentiel $\left(\mathrm{TC}_{\mathrm{DGPS}}\right)$, lorsque ces données étaient disponibles et concomitantes.

\section{Résultats}

La figure 3 présente les quatre différents traits de côte définis précédemment sur le site

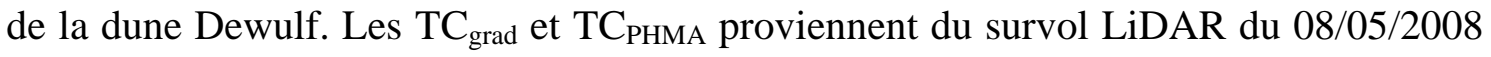
tandis que le $\mathrm{TC}_{\mathrm{DGPS}}$ a été réalisé deux semaines plus tôt, le 25/04/2008. Nous ne disposons malheureusement pas de photographie aérienne de la même année, le cliché le plus proche dans le temps datant du 09/06/2009. Malgré un recul de $2 \mathrm{~m}$ entre 2008 et 2009 mesuré par levés topographiques au DGPS, nous pouvons constater que les $\mathrm{TC}_{\text {grad }}$ et $\mathrm{TC}_{\mathrm{DGPS}}$ se confondent pratiquement avec le trait de côte issu de l'orthophographie. Seul le $\mathrm{TC}_{\text {pHмa }}$ se situe bien en avant par rapport au pied de dune, ceci étant communément observé sur d'autres littoraux dunaires (e.g., BRETEL et al., 2013). Cette comparaison montre clairement qu'un trait de côte défini par le niveau des plus hautes mers astronomiques ne correspond pas nécessairement à la position réelle de la position du trait de côte défini sur la base de critères géomorphologiques. De plus, cette comparaison permet de montrer la pertinence de l'extraction du pied de dune par la méthode du gradient de pente. En effet, les $\mathrm{TC}_{\text {grad }}$ et $\mathrm{TC}_{\mathrm{DGPS}}$ sont, dans la très grande majorité des cas, proches l'un de l'autre, l'écart moyen calculé entre les deux étant de l'ordre de $2 \mathrm{~m}$ (écart-type de $1,5 \mathrm{~m}$ ).

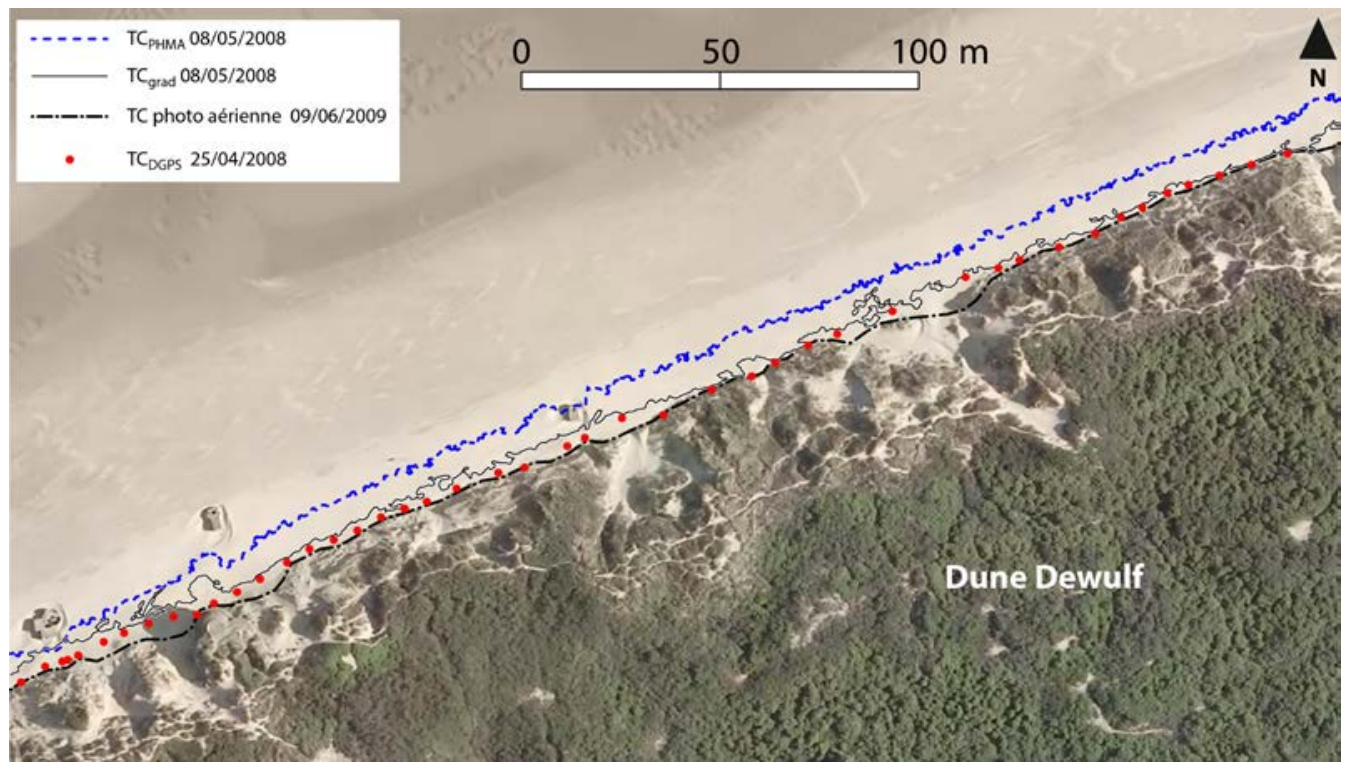

Figure 3. Comparaison des différents traits de côte pour le secteur de la dune Dewulf à l'est de Dunkerque (orthophotographie IGN de 2009). 
La variabilité spatiale des différences de position entre le $\mathrm{TC}_{\text {grad }}$ et le $\mathrm{TC}_{\mathrm{PHMA}}$ a également été analysée. L'examen de l'évolution des deux différents traits de côte entre 2008 et 2011 sur les sites du platier d'Oye et des dunes Dewulf -Marchand (figure 4) montre des évolutions similaires dans la plupart des secteurs (i.e., avancée ou recul des deux indicateurs de trait de côte), mais on constate aussi des évolutions différentes à certains endroits. C'est le cas notamment dans la partie centrale du platier d'Oye entre

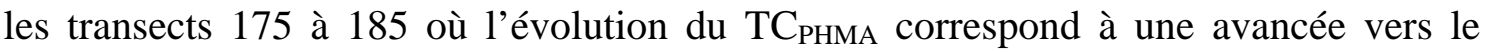
large, alors que la position du $\mathrm{TC}_{\text {grad }}$ varie peu, ce qui suggère une stabilité de la ligne de côte (figure 4A).
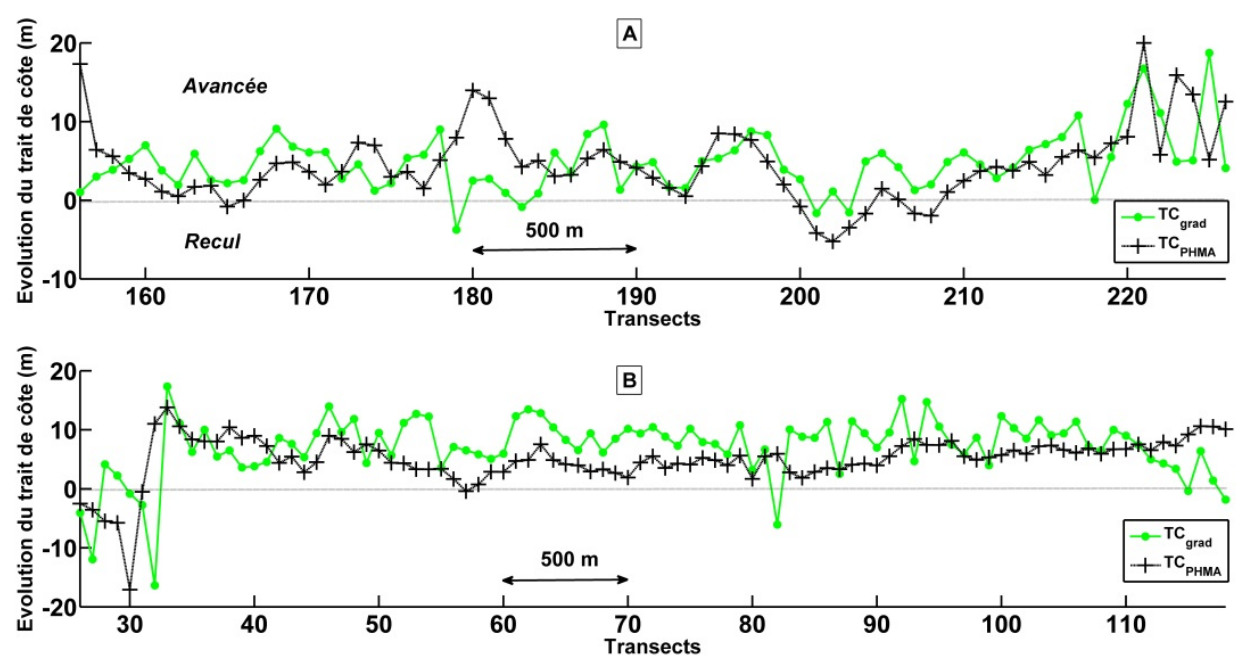

Figure 4. Evolution des $T C_{P H M A}$ et $T C_{\text {grad }}$ entre 2008 et 2011 sur les sites (A) du platier d'Oye et (B) des dunes Dewulf et Marchand.

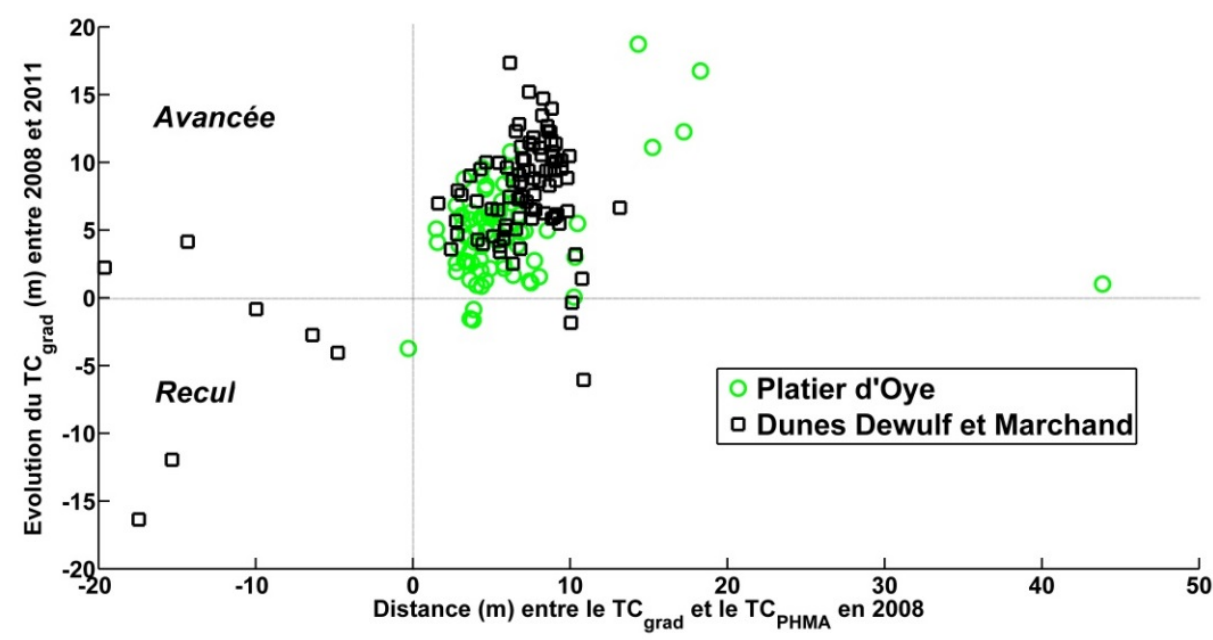

Figure 5 Relation entre la distance initiale $T D_{\text {grad }}-T C_{P H M A}$ et l'évolution $d u T C_{\text {grad }}$ entre 2008 et 2011 sur les sites du platier d'Oye et des dunes Dewulf et Marchand. 


\section{XIII ${ }^{\text {èmes }}$ Journées Nationales Génie Côtier - Génie Civil \\ Dunkerque, 2-4 juillet 2014}

La figure 5 montre la relation qui existe entre la distance initiale entre le $\mathrm{TC}_{\text {grad }}$ et le TC PнмA $_{\text {en }} 2008$ et l'évolution du $\mathrm{TC}_{\text {grad }}$ entre 2008 et 2011, une valeur négative de distance $\mathrm{TC}_{\text {grad }}$ - $\mathrm{TC}_{\text {PHMA }}$ signifiant que le $\mathrm{TC}_{\mathrm{PHMA}}$ est situé à l'arrière du $\mathrm{TC}_{\text {grad, }}$ ce qui correspond à une absence quasi-totale de haut de plage à marée haute. La différence de distance entre le $\mathrm{TC}_{\text {grad }}$ et le $\mathrm{TC}_{\mathrm{PHMA}}$ est positive dans la plupart des cas, ce qui montre que le $\mathrm{TC}_{\text {PHмA }}$ est généralement situé à l'avant du $\mathrm{TC}_{\text {grad. }}$ L'analyse de l'évolution de la position du $\mathrm{TC}_{\text {grad }}$ révèle en outre que lorsque le $\mathrm{TC}_{\text {PHMA }}$ est situé à l'avant du $\mathrm{TC}_{\text {grad, }}$ le trait de côte a été stable ou a connu une avancée associée à un développement ou une progradation des dunes côtières dans la très grande majorité des cas. A l'inverse, lorsque le $\mathrm{TC}_{\text {PHMA }}$ est situé à l'arrière du $\mathrm{TC}_{\text {grad }}$ (valeur négative), le trait de côte a plutôt eu tendance à reculer, bien que l'on puisse aussi observer quelques cas d'avancée de la position du $\mathrm{TC}_{\text {grad. }}$.

\section{Conclusion}

L'extraction du trait de côte par la méthode du gradient de pente ( $\left.\mathrm{TC}_{\text {grad }}\right)$ a montré une bonne correspondance avec la position du trait de côte mesurée in situ par DGPS ( $\left(\mathrm{TC}_{\mathrm{DGPS}}\right)$. Le $\mathrm{TC}_{\mathrm{PHMA}}$, qui correspond au niveau marégraphique des plus hautes marées astronomiques, montre une moins bonne correspondance avec le $\mathrm{TC}_{\mathrm{DGPS}}$. Le $\mathrm{TC}_{\text {PHMA }}$, correspondant à un niveau d'eau maximum théorique, mais sans prendre en compte d'éventuelles surcotes ou décotes, il en résulte communément des différences entre sa position sur le haut de plage et la limite morphologique de la ligne de rivage. Il peut se situer soit à l'arrière, soit à l'avant du $\mathrm{TC}_{\text {grad, }}$,e qui est majoritairement le cas sur les sites couverts par cette étude. Les mesures de l'évolution du trait de côte ont montré que lorsque le $\mathrm{TC}_{\mathrm{PHMA}}$ est situé à l'avant du $\mathrm{TC}_{\text {grad, }}$ le trait de côte a généralement connu une avancée vers le large pendant les années récentes en raison de la progradation du pied de dune. Une position vers le large du $\mathrm{TC}_{\text {РнмА }}$ par rapport au $\mathrm{TC}_{\text {grad }}$ correspond à une importante largeur du haut de plage, ce qui reflète un stock sédimentaire conséquent à l'origine de la progression du trait de côte vers le large. A contrario, les secteurs dunaires où le $\mathrm{TC}_{\mathrm{PHMA}}$ se confond avec le $\mathrm{TC}_{\text {grad }}$ ou est situé à l'arrière de celui-ci correspondent à des secteurs où les dunes côtières sont vulnérables face à d'éventuels hauts niveaux d'eau qui peuvent venir éroder le pied de dune et provoquer un recul du trait de côte. La position du TC $\mathrm{P}_{\text {pHM }}$ par rapport au $\mathrm{TC}_{\text {grad }}$, qui constitue une indication de la largeur du haut de plage, pourrait donc représenter un bon indicateur de la tendance évolutive de la ligne de rivage le long de littoraux dunaires.

\section{Références}

AUBRY A., RUZ M.H. (2008). Influences des épis sur la morphodynamique d'une plage à barres et bâches, nord de la France. Zeitschrift für Geomorphologie, Vol. 52, pp 146-166. 
ANTHONY E.J., HEQUETTE A. (2007). The grain size characterisation of coastal sand from the Somme estuary to Belgium: sediment segregation processes and sources consideration. Sedimentary Geology, 202, pp 369-382. http://dx.doi.org/10.1016/.sedgeo.2007.03.022 BOAK E.H., TURNER I.L. (2005). Shoreline definition and detection: A review. Journal of Coastal Research, Vol. 21, pp 688-703. http://dx.doi.org/10.2112/03-0071.1

BRETEL P., FROIDEVAL L., MONFORT O., LEVOY F. (2013). Coastline identification from airborne LiDAR surveys: a multi criteria approach. Coastal Dynamics 2013, Arcachon, pp 213-224.

BROCK J.C., PURKIS S.J. (2009). The emerging role of Lidar remote sensing in coastal research and resource management. Journal of Coastal Research, Sp. Issue 53, pp 1-5.

DERONDE B., HOUTHUYS R., HENRIET J.P., VAN LANCKER V. (2008). Monitoring of the sediment dynamics along a sandy shoreline by means of airborne hyperspectral remote sensing and LiDAR: a case study in Belgium. Earth Surface Processes and Landforms, Vol. 33, pp 280-294. http://dx.doi.org/10.1002/esp.1545

HEQUETTE A., AERNOUTS D. (2010). The influence of nearshore sand bank dynamics on shoreline evolution in a macrotidal coastal environment, Calais, Northern France. Continental Shelf Research, Vol. 30, pp 1349-1361. http://dx.doi.org/10.1016/j.csr.2010.04.017

LEVOY F., ANTHONY E.J., MONFORT O., ROBIN N., BRETEL P. (2013). Formation and migration of transverse bars along a tidal sandy coast deduced from multi-temporal Lidar datasets. Marine Geology, Vol. 342, pp 39-52. http://dx.doi.org/10.1016/j.margeo.2013.06.007

MASPATAUD A., RUZ M.H., HEQUETTE A. (2011). Storm-driven shoreline evolution on a macrotidal coast: short- to medium-term spatial variability. A case study on the northern coast of France. Coastal Sediments '11, Miami, pp. 927-940.

RUZ M.-H., HEQUETTE A., MASPATAUD A. (2009). Identifying forcing conditions responsible for foredune erosion on the northern coast of France. Journal of Coastal Research, Sp. Issue 56, pp 356-360.

SIPKA V., ANTHONY E.J. (1999). Morphology and hydrodynamics of a macrotidal ridge and runnel beach under modal low wave conditions. Journal de Recherche Océanographique, Vol. 24, pp 25-31.

STEPHAN P., SUANEZ S. (2004). Dynamique morphosédimentaire des cordons dunaires de Trétuder et de Saint-Michel-en-Grève (baie de Saint-Michel-en-Grève Côtes d'Armor) entre 1990 et 2003. Bulletin de l'Association de Géographes Français, Vol. 81, pp 438-448.

STOCKDON H., DORAN K., SALLENGER A. (2009). Extraction of LiDAR-based dune-crest elevations for use in examining vulnerability of beaches to inundation during hurricanes. Journal of Coastal Research, Sp. Issue 53, pp 59-65. http://dx.doi.org/10.2112/SI53-007.1 